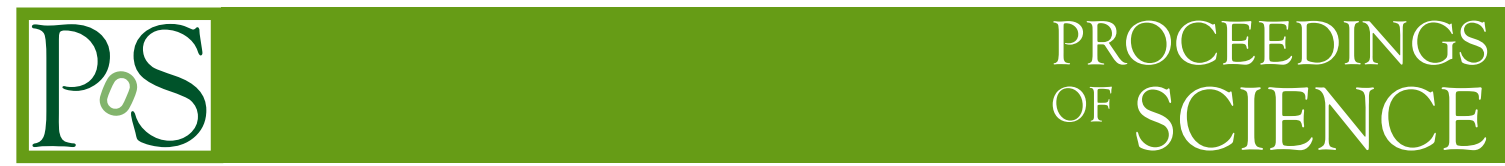

\title{
The KMI phi project
}

\author{
Yasumichi Aoki * \\ Kobayashi-Maskawa Institute for the Origin of Particles and the Universe (KMI), Nagoya \\ University \\ E-mail: yaoki@kmi.nagoya-u.ac.jp
}

One of the major scientific missions that KMI promotes is the computational research for the physics beyond the standard model. The high performance parallel computer $\varphi$ was installed at KMI in early 2011 for that purpose. Lots of efforts have been put together for the operation and the utilization of $\varphi$ since then. LatKMI collaboration leads the flagship project that have been using a large fraction of $\varphi$, exploring the possibility of composite description of the Higgs particle fonud in LHC experiment. Their scientific achievements will be briefly discussed.

KMI International Symposium 2013 on "Quest for the Origin of Particles and the Universe" 11-13 December, 2013

Nagoya University, Japan

${ }^{*}$ Speaker. 


\section{Introduction}

The Kobayashi-Maskawa Institute for the Origin of Particles and the Universe (KMI) has been established in 2010. One of the main objectives is to explore new frontiers of modern physics beyond the Standard Model. One last and missing piece of the standard model of particle physics at that moment was the Higgs particle, which would appear as a remnant of the Higgs mechanism for spontaneous breakdown of the electroweak gauge symmetry. Unsatisfactory property of the standard model had been not only this "missing" fact, but also the hierarchy problem, where "unnatural" fine-tuning would be required to have Higgs boson mass at the electroweak scale $(\sim 100$ $\mathrm{GeV}$ ). As such, studying the standard model and models beyond that from the mechanism of the electroweak symmetry breaking has been one of the main subjects of the research in elementary particle physics. The situation has not been changed by the discovery of the missing particle, the Higgs.

One of the attractive theories for the new physics, which should amend the problem of the standard model, is technicolor. The theory, which is explained in more detail in later, mimics QCD and the the Higgs boson would appear as a composite state like quark-antiquark bound state in QCD. Due to the inherent strong coupling nature, the theory has not been fully tested and the difficulty of analysis associated it seems to have contributed to "less-popularity" compared with the much popular extension of the standard model, supersymmetry. The situation has been changing due to the progress in computer and computational method reinforced in the development in QCD in the standard model.

The establishment of KMI took place while the growing interest of this new strong dynamics in the lattice QCD community emerged. A high performance computer system, named $\varphi$ and dedicated for such purpose, was installed then and has been fully running to produce various interesting outcomes since then. In the following sections, operation and utilization of $\varphi$ as well as the related systems are described, and the scientific meaning of the outcomes mainly obtained by the LatKMI collaboration are reviewed.

\section{KMI computational resources for theoretical studies}

In the KMI Center for Theoretical Studies, computer systems with various scales and purposes were installed to accelerate the researches based on numerical efforts. The central system is $\varphi$, where the large scale computations for the theories beyond the standard model of particle physics are performed. The JLDG system, a global grid storage system, installed in KMI, allows the researchers to move the massive data from $\varphi$ to another site in Japan or world, and vice versa. Other small scale cluster computers are also installed to enhance the numerical capabilities of KMI. All of these, which are explained in detail below, are managed by the Computational Theoretical Physics Laboratory in KMI.

\subsection{KMI high performance computer $\varphi$}

A high performance parallel computer system was installed in KMI and started its operation on March 2nd 2011. This was and is the only large computer system in the world, to our knowledge, dedicated to the study of the theoretical particle physics for the search of the physics beyond the 
standard model. The purpose is to face the challenges in the elementary particle physics, namely to solve the non-perturbative dynamics of quantum gauge field theory, aiming to elucidate the origin of the mass in elementary particles from the dynamical mechanism. The lattice gauge theory, a rigorous non-perturbative definition of the gauge theory, is used to numerically tackle this challenging problem.

The system, assembled by NEC, consists of 148 nodes equipped with dual Intel Xeon 5680 CPU with 6 cores (HP ProLiant SL2x170z G6) and 23 nodes with dual Intel Xeon 5670 CPU with 6 cores and 3 NVIDIA Tesla M2050 GPGPUs (HP ProLiant SL390s G7). These computation nodes, the high performance disk system with Lustre file system (DDN SFA10000), front-end node and other managing nodes are interconnected with a Voltaire QDR InfiniBand switch. The theoretical peak performance of the whole system counts 62TFlops, which made this system one of the fastest computers in the central Japan area at the installation time.

The computer system was named $\varphi$ after the following Greek words: the name of our prefecture "Aichi": $\varphi \imath \lambda o \sigma o \varphi \imath \alpha$, "Physics": $\varphi v \sigma \imath \kappa o \zeta$, the complex phase " $\varphi$ " as a symbol of the Kobayashi-Maskawa theory, and the initial letter of the director general Maskawa in one version of his signature : $\tau . \varphi \kappa \alpha \omega \alpha$.
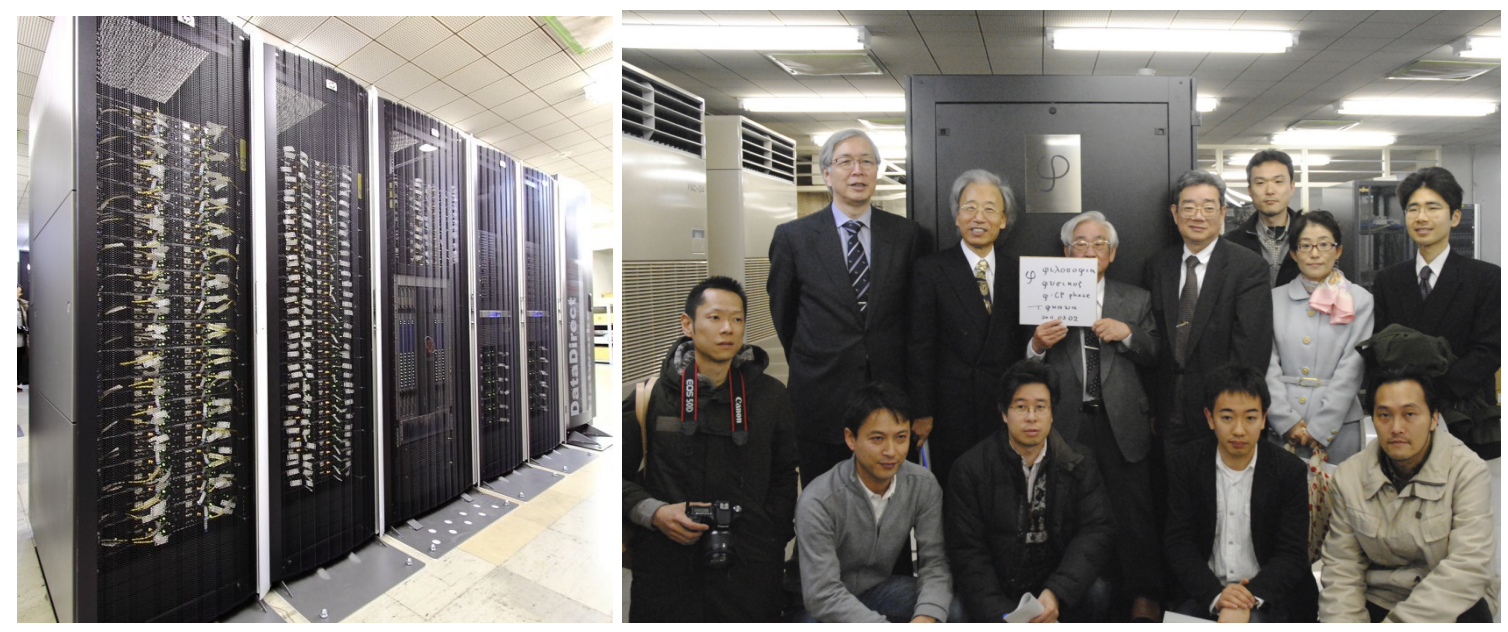

Figure 1: The $\varphi$ cluster computing system installed at KMI (left). $\varphi$ with the director general Maskawa with people including KMI staff at the inauguration ceremony on March 2nd, 2011 (right).

\subsection{JLDG}

Japan Lattice Data Grid (JLDG) ${ }^{1}$ is a global data grid server for the Japanese community utilizing large-scale numerical computation with lattice field theory. The servers at Nagoya site was installed through the support by HPCI (High Performance Computing Infrastructure) Strategic Program Field 5, and has been up and running since March 2012. Massive data once generated on $\varphi$ can be ported to another site in Japan through JLDG, which connects all the main sites conducting lattice efforts through SINET $^{2}$ VPN. The system also serves as robust back up facility of

\footnotetext{
${ }^{1}$ http://www.jldg.org/

${ }^{2}$ http://www.sinet.ad.jp/
} 
gauge configurations with replica files always being made automatically at different servers in geographically separated cites. JLDG also serves as cluster of servers of the International Lattice Data Grid (ILDG) [1] for the lattice community in the world for sharing precious quantum ensembles of gauge field configurations generated in large-scale computations.

\subsection{Small scale clusters}

Upon the establishment of KMI another cluster computing system was installed for the research in the area of cosmology and theoretical astrophysics. This cluster system, named COSMOS, consisted of 28 computational nodes with dual 4-core Intel Xeon. COSMOS, later supplemented with 4 nodes having dual 6-core Intel Xeon, has been fully utilized for the KMI mission at Computational Theoretical Physics Laboratory in collaboration with the Division of Cosmology and Theoretical Astrophysics. Some results using this system is reported in these proceedings by Hikage [2].

Two subsystems of $\varphi$ were installed in KMI after the start of the successful operation of $\varphi$ to further enhance the computational capability and to meet the growing demand in computations for lattice gauge theory as well as hydrodynamics computations for quark-gluon plasma and numerical analysis in cosmology. One, named ctpl1 has 25 computation nodes, having similar composition with the non-GPGPU part of $\varphi$. Newer machine, ctpl2, is equipped with 36 nodes of dual Intel Xeon E5-2670 (8 core) and 2 nodes with dual Intel Xeon Phi 5110P. Outcome of these machines are reported by Hikage [2], Nonaka [3], and summarized in Sect. 5 for researches in lattice gauge theory.

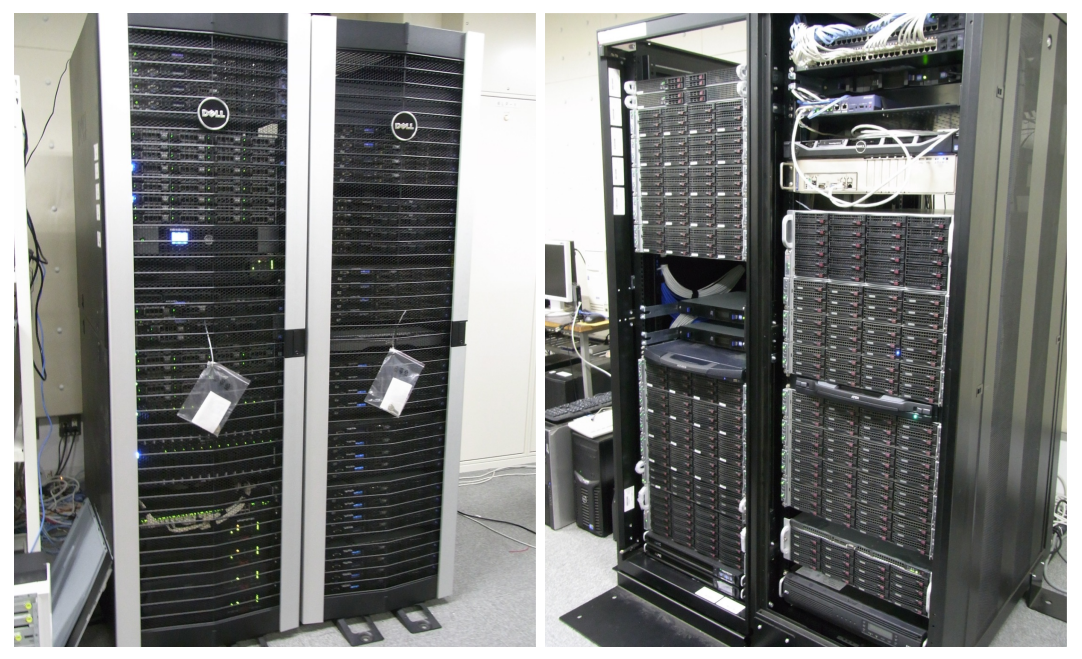

Figure 2: The cluster computer system COSMOS for cosmology and theoretical astrophysics (left), the $\varphi$ subsystems ctpl1 and ctpl2 (right).

\section{Operation of $\varphi$}

Operation and utilization of $\varphi$ are among the most important tasks of the Computational Theoretical Physics Laboratory (CTPL) in KMI. Along the operation, proposals of the projects are gath- 
ered from PIs in KMI, Department of Science, Department of Mathematics, or Solar-Terrestrial Environmental Laboratory in Nagoya University. In each project PI can appoint members whose affiliation does not have any restriction: members can even be outside of Nagoya University.

Project proposals are evaluated in the $\varphi$ working group (WG), which is formed mainly by members in CTPL: Y. Akamatsu, ${ }^{3}$ Y. Aoki, ${ }^{3}$ T. Aoyama, ${ }^{3}$ K. Miura, ${ }^{4}$ K. Nagai, ${ }^{5}$ C. Nonaka, ${ }^{3}$ M. Tanabashi, ${ }^{3}$ and T. Yamazaki. ${ }^{6}$ The WG deals with the administrative and practical issues arising in the operation. The decision made in the WG is passed to the steering committee of CTPL for approval. Among the works dealt by the WG, making the policy, the review and screening of the projects which takes place just before the operation term starts are the most important tasks.

Since the test use period (March-May 2011) concluded, operations with a term fixed for six months have been taken place. In each operation term up to four projects which matches the KMI missions and requires large computation are accepted as "priority projects" which are given a higher priority in the operation. This is for an efficient use of the large computer, otherwise it could end up letting many projects with each share being far from optimal. So far three projects led by LatKMI members, as well as one project led by Masahi Hayakawa, ${ }^{7}$ are accepted for the promotion in each term.

\section{Strong dynamics to replace the standard-model Higgs sector}

Masses of the elementary particles are linked with a breaking of the electroweak gauge symmetry. In the standard model the breaking results from the Higgs mechanism. For long time this mechanism has been under serious scrutiny and has been one of the central problem in particle physics. The issue is that unnatural tuning of the parameter is needed to stabilize this mechanism. Supersymmetry has been the most popular extension of the standard model to cope with the stability. Among other possible solutions an intriguing possibility is provided by technicolor [4], where the gauge symmetry breaking is caused by the spontaneous breaking of the chiral symmetry in the strongly coupled techni-sector. ${ }^{8}$

In the standard-model Higgs sector an isodoublet Higgs, which has four real degree of freedom, developing a scalar condensate (due to negative mass squared in the Lagrangian) and the remaining three degree of freedom become Nambu-Goldstone (NG) modes, which are absorbed to the weak gauge bosons $\left(W^{ \pm}, Z^{0}\right)$ to make them massive (Higgs mechanism). The problem of this model is the power divergence in the large energy limit, which makes the Higgs boson mass extremely sensitive to the details of high energy structure of the fundamental law of the nature. That leads to an aforementioned fine-tuning problem.

In technicolor models hypothetical new particles, techni-quarks, which interact with each other by exchanging techni-gluons, mimicking the strong dynamics of quarks in QCD in the standard model, make scalar bilinear condensate, which result in the spontaneous break-down of the chiral

\footnotetext{
${ }^{3}$ CTPL, KMI Center for Theoretical Studies, Nagoya University

${ }^{4}$ CTPL, KMI Center for Theoretical Studies, Nagoya University since April 2013

${ }^{5} \mathrm{KMI}$, Nagoya University

${ }^{6}$ CTPL, KMI Center for Theoretical Studies, Nagoya University until March 2014

${ }^{7}$ Department Physics, Nagoya University

${ }^{8}$ For a review, see [5].
} 
symmetry. Resulting NG bosons, techni-pions, once the techni-sector is replaced with the Higgs sector in the standard model, play the same role as the NG modes in the standard-model Higgs and make the weak gauge bosons massive. Because no fundamental scalar enters in this theory, there is no power divergence (only logarithmic divergence exists), thus no extreme sensitivity to the high scales. Counterpart of the Higgs boson would be a composite scalar particle due to the strong dynamics. The mass of the "Higgs" boson at the electroweak scale will naturally results in the dynamical scale set by the decay constant of the techni-pions, which works as the counterpart of the Higgs condensate and thus needs to be $O(100) \mathrm{GeV}$.

In the standard-model quarks and charged leptons acquire masses from the Yukawa interaction with the Higgs field which develops the condensate. In technicolor models, so-called extended technicolor (ETC) provides a dynamical mechanism to produce the masses through four-Fermi interactions (two standard-model fields and two techni-fields) induced through a gauge symmetry breaking in the extended sector. However, at the same time, four-Fermi interaction made exclusively with standard-model fields can be induced and easily violate the experimental limit of the flavor changing neutral current (FCNC). One can set the scale of the ETC to be large enough to suppress this effect because the induced four-Fermi interaction accompanies $1 / \Lambda_{E T C}^{2}$ (inverse ECT scale squared). However, this can make the standard-model fermion masses too small.

A work around of this problem is provided by the walking technicolor model [6], [7, 8, 9] where the gauge coupling runs very slowly (thus walking) and anomalous dimension of the mass operator is as large as $\gamma \sim 1$ at the walking region. These features, extraordinary from QCD in the standard model, expected to realize a sizable fermion mass with the FCNC sufficiently suppressed. Such a theory may be found by increasing the number of mass-less fermions $\left(N_{f}\right)$ in QCD $\left(N_{f}=2\right.$ in the standard model), close to the fictitious phase boundary which separates the phase with broken chiral symmetry ( $\operatorname{small} N_{f}$ ) and conformal phase (larger $N_{f}$ ).

As the theory of interest has a strongly interacting nature, analytic studies limit the reach of the physical intent. Success of the numerical approach with lattice gauge theory for QCD in the standard model indicates the good use of the same machinery for the purpose here. Indeed, with pioneering works starting around early 90th discussing the phase boundary [10, 11, 12], the field has been growing and has become a one of the major fields in the community of lattice field theory (see the contribution by Kuti in these proceedings [13]) and has shown its potential power.

Earlier lattice studies are concentrating on figuring out where the phase boundary in $N_{f}$ space is. Eventually some works are focusing on the properties of the walking (slow running and large mass anomalous dimension), and a few dealing with oblique electroweak correction, Peskin-Takeuchi $S$ parameter [14, 15]. These properties govern the fate of a particular model in a indirect way. As the LHC unfolded the "new" particle, which turned out to be consistent with the standard-model Higgs and its mass is $125 \mathrm{GeV}$, there emerged another, somewhat more direct target to test the models. That is if the particular model can produce such a light composite state, as a typical composite spectrum is expected to lie above $\sim 1 \mathrm{TeV}$.

\section{LatKMI study of strong dynamics}

LatKMI collaboration, formed after the establishment of KMI, began with most members residing in KMI. The collaboration was aiming to study the many flavor nature of QCD using 
lattice gauge theory and massive use of the KMI high-performance computer $\varphi$.

There can be various definitions of the lattice theory to express the single continuum theory in the limit where the lattice spacing $a$ is driven to vanish $a \rightarrow 0$. LatKMI collaboration uses the Highly Improved Staggered Quark (HISQ) action [16] for the strongly interacting fermions. HISQ is one of the staggered fermion formulation, where the $\mathrm{U}(1)_{V}$ baryon number, taste-flavor diagonal axial (U(1)-degree-of-freedom), and $\mathrm{SU}\left(N_{f} / 4\right)_{L} \times \mathrm{SU}\left(N_{f} / 4\right)_{R}$ flavor-chiral symmetry $\left(N_{f}=4 n\right.$ with $n \leq 2$ case) are exact even at non-zero lattice spacing $a$, while the full $\mathrm{U}\left(N_{f}\right)_{L} \times \mathrm{U}\left(N_{f}\right)_{R}$ is expected to be recovered in the continuum limit $(a \rightarrow 0)$. There is a flavor violation at $a \neq 0$ and the HISQ action is tailored to minimize them in a way of Symanzik effective theory construction. The exact symmetry of a part of the flavors and the HISQ improvement yield a correct count of the light degree of freedom, which is crucial for the interested chiral dynamics.

LatKMI makes use of the good properties of HISQ and systematically studies the $N_{f}$ dependence of the dynamics of SU(3) gauge theory for $N_{f}=4,8,12$ and 16. $N_{f}=4$ is regarded as a reference of a theory with spontaneous chiral symmetry breaking ( $\mathrm{S} \chi \mathrm{SB}$ ) and $N_{f}=16$ is for a conformal theory known from perturbation theory. $N_{f}=8$ and 12 are in the prime region of interest. The main observables are the hadronic spectrum in the mass deformed theory, which is analyzed against the $\mathrm{S} \chi \mathrm{SB}$ and conformal scenarios. Fermion mass $m_{f}$ and the system volume $L^{3} \times T$ are used for the handles to see the property of the system.

The initial results as of 2011 were summarized in Ref. [17]. Here in the following the updates are reported.

\section{1 $N_{f}=12$ QCD}

With a global survey of the parameter space two values of bare gauge coupling are selected. For each value of gauge coupling some wide range of $m_{f}$ and $L$ are examined. Mass of the composite $\pi$ and $\rho$ states $\left(m_{\pi}\right.$ and $\left.m_{\rho}\right)$ as well as the pion decay constant $\left(f_{\pi}\right)$ obtained are consistent with conformal type scaling (so-called hyperscaling) and the mass anomalous dimension $\gamma$ obtained through the scaling $m_{H} \sim m_{f}^{1 /(1+\gamma)}$ is consistent among the different observables, which is in the range of $0.4 \lesssim \gamma \lesssim 0.5$. Multiple values of gauge coupling allow one to study the scaling towards the continuum limit of the mass deformed theory, which ensures the simulation parameters are in the proper range. The results are published in [18] and updated results are presented in the contribution by Ohki in these proceedings [19].

\section{$5.2 N_{f}=8$ QCD}

Similar global survey as $N_{f}=12$ leads to an optimal choice of bare gauge coupling and some wide range $m_{f}$ and $L$ are examined. In this case the composite spectra are consistent with both $\mathrm{S} \chi \mathrm{SB}$ and conformal type scaling. In the conformal type scaling the mass anomalous dimension is not universal among different observables but roughly $\gamma \sim 1$ (as large as one hope for the walking technicolor theory). This kind of property is not found in $N_{f}=4$ or $N_{f}=12$. As $m_{f}$ is driven more toward the chiral limit, either scenario will eventually fail. If the S $\chi \mathrm{SB}$ scenario remains and the conformal scenario fails in that limit, then what is seen now is what is expected for the walking technicolor theory, where the quark mass $m_{f}$ acts as the probe to scan the renormalization scale. Along with the $\mathrm{S} \chi \mathrm{SB}$ scenario one needs to first estimate the pion decay constant in the chiral 
limit which sets the scale of the technicolor theory. The techni-rho meson mass, which is not quite sensitive to the detail of models, is measured and the ratio $m_{\rho} / f_{\pi}$ is given as a prediction. The results are published in [20] and a digest is given in the contribution by Nagai in these proceedings [21].

\subsection{Flavor singlet scalar in $N_{f}=8$ and 12 QCD}

After the discovery of "Higgs" particle in LHC experiments, every theory to extend the standard model is required to allow the existence of $125 \mathrm{GeV}$ Higgs-like scalar particle. A flavor singlet scalar $\sigma$ could be a Higgs impostor in technicolor theories. Although a typical composite mass will be larger than $\sim 1 \mathrm{TeV}$ for composite models, it can be much lighter if the dilatonic nature is intact for the walking technicolor theory [6]. One can test this light "Higgs" possibility in lattice QCD computations model-independently from the first principles.

Study of flavor singlet sector in lattice numerical computation is known to be a challenging task, as it requires noisy measurements originating from direct impact of the gauge field fluctuation. Using a very high statistics by fully utilizing $\varphi$ and a variance reduction method for staggered fermions, LatKMI first examined the $\sigma$ spectrum in $N_{f}=12$ theory. As summarized in Sect. 5.1 it did not turn out to be a good candidate of walking technicolor, but the study may provide a useful information. Indeed, a novel spectral property where the flavor singlet scalar $\sigma$ appears lighter than $\pi: m_{\sigma}<m_{\pi}$ for a wide range of input quark mass studied. This kind of special property has been seen in SU(2) gauge theory with two adjoint fermion number in the glueball sector [22]. But, this is the first time to observe in SU(3) gauge theory and with a large overlap with the fermionic bilinear operator. This suggests a possibility of realizing a light scalar composite in a near-by theory, $N_{f}=8$ which shares a conformal type property with $N_{f}=12$. The results are published in [23] and reported in these proceedings by Ohki [19].

Triggered by the success of $N_{f}=12$ theory in finding a novel spectrum, LatKMI extended the study of flavor singlet scalar to $N_{f}=8$ theory. The $\sigma$ turns out to be as light as $\pi$ for a wide range of input quark mass studied, being similar property as $N_{f}=12$ as expected. This indicates a possibility of having a composite Higgs as light as $125 \mathrm{GeV}$. But, further higher precision is required to see the fate of this theory. The results are published in [24] and reported in these proceedings by Yamazaki [25].

\subsection{Other topics}

Other than those reported above, there are also on-going projects in LatKMI collaboration. The Peskin-Takeuchi $S$ parameter is an important quantity to calculate in a candidate theory of composite Higgs. A method for the staggered fermions are developed, tested and are being used for systematic study on the gauge configurations generated already for spectrum. Finite temperature nature is important for a candidate theory of composite Higgs, as it may open a possibility of having first order electroweak phase transition for a solution to the Baryogenesis. Topological insight is interesting as well for another handle to study the conformal type scaling as well as the chiral anomaly. A new technique, Yang-Mills gradient flow (Wilson/Symanzik flow) [26] is used for that purpose. 


\section{Conclusion}

KMI high performance computer $\varphi$ has been fully utilized since its installation in March 2011, and has produced interesting results concerning the (near) conformal nature of the dynamics of gauge theory coupled with a large number of massless fermions. Results obtained by LatKMI collaboration suggest a possibility of having Higgs like particle as a composite state made of strong dynamics in near-conformal region. Solidness of this emerging picture will have to be investigated further and the precision needs to be improved. However, the initial important step has been made and surely the KMI effort has marked its footprints in this attractive direction of exploration of the physics beyond the standard model.

\section{References}

[1] M. G. Beckett, B. Joo, C. M. Maynard, D. Pleiter, O. Tatebe, et. al., Building the International Lattice Data Grid, Comput.Phys.Commun. 182 (2011) 1208-1214, [arXiv: 0910.1692 ].

[2] C. Hikage, Unveiling cosmic structure formation with galaxy imaging and redshift surveys, PoS KMI2013 (2015) 021.

[3] C. Nonaka, Relativistic hydrodynamics in high-energy heavy ion collisions, PoS KMI2013 (2015) 025 .

[4] S. Weinberg, Implications of Dynamical Symmetry Breaking, Phys.Rev. D13 (1976) 974-996.

[5] E. Farhi and L. Susskind, Technicolor, Phys.Rept. 74 (1981) 277.

[6] K. Yamawaki, M. Bando, and K.-i. Matumoto, Scale Invariant Technicolor Model and a Technidilaton, Phys.Rev.Lett. 56 (1986) 1335.

[7] T. Akiba and T. Yanagida, Hierarchic Chiral Condensate, Phys.Lett. B169 (1986) 432.

[8] T. W. Appelquist, D. Karabali, and L. Wijewardhana, Chiral Hierarchies and the Flavor Changing Neutral Current Problem in Technicolor, Phys.Rev.Lett. 57 (1986) 957.

[9] B. Holdom, Techniodor, Phys.Lett. B150 (1985) 301.

[10] Y. Iwasaki, K. Kanaya, S. Sakai, and T. Yoshie, Quark confinement and number of flavors in strong coupling lattice QCD, Phys. Rev. Lett. 69 (1992) 21-24.

[11] F. R. Brown, H. Chen, N. H. Christ, Z. Dong, R. D. Mawhinney, et. al., Lattice QCD with eight light quark flavors, Phys.Rev. D46 (1992) 5655-5670, [hep-lat/9206001].

[12] P. Damgaard, U. M. Heller, A. Krasnitz, and P. Olesen, On lattice QCD with many flavors, Phys.Lett. B400 (1997) 169-175, [hep-lat/9701008].

[13] J. Kuti, The Higgs particle and the lattice, PoS KMI2013 (2015) 002.

[14] M. E. Peskin and T. Takeuchi, A New constraint on a strongly interacting Higgs sector, Phys. Rev. Lett. 65 (1990) 964-967.

[15] M. E. Peskin and T. Takeuchi, Estimation of oblique electroweak corrections, Phys. Rev. D46 (1992) 381-409.

[16] HPQCD Collaboration, E. Follana et. al., Highly Improved Staggered Quarks on the Lattice, with Applications to Charm Physics, Phys. Rev. D75 (2007) 054502, [hep-lat / 0610092 ]. 
[17] LatKMI Collaboration, Y. Aoki, T. Aoyama, M. Kurachi, T. Maskawa, K.-i. Nagai, H. Ohki, A. Shibata, K. Yamawaki, and T. Yamazaki, The KMI Lattice Project - Exploring for Technicolor from $Q C D$, . in Proceedings of KMI Inauguration Conference on Quest for the Origin of Particles and the Universe (KMIIN) : Nagoya, Japan, October 24-26, 2011, Y. Aoki, K. Hayasaka, T. Iijima, T. Maskawa, M. Tanabashi, and K. Yamawaki ed., World Scientific (2013).

[18] LatKMI Collaboration, Y. Aoki, T. Aoyama, M. Kurachi, T. Maskawa, K.-i. Nagai, H. Ohki, A. Shibata, K. Yamawaki, and T. Yamazaki, Lattice study of conformality in twelve-flavor QCD, Phys.Rev. D86 (2012) 054506, [arXiv:1207.3060].

[19] LatKMI Collaboration, Y. Aoki, T. Aoyama, M. Kurachi, T. Maskawa, K. Miura, K.-i. Nagai, H. Ohki, E. Rinaldi, A. Shibata, K. Yamawaki, and T. Yamazaki, Conformal dynamics in Nf=12 lattice QCD, PoS KMI2013 (2015) 004.

[20] LatKMI Collaboration, Y. Aoki, T. Aoyama, M. Kurachi, T. Maskawa, K.-i. Nagai, H. Ohki, A. Shibata, K. Yamawaki, and T. Yamazaki, Walking signals in $N f=8 Q C D$ on the lattice, Phys.Rev. D87 (2013) 094511, [arXiv:1302.6859].

[21] LatKMI Collaboration, Y. Aoki, T. Aoyama, M. Kurachi, T. Maskawa, K. Miura, K.-i. Nagai, H. Ohki, E. Rinaldi, A. Shibata, K. Yamawaki, and T. Yamazaki, Walking signals in eight flavor QCD on the lattice, PoS KMI2013 (2015) 005.

[22] L. Del Debbio, B. Lucini, A. Patella, C. Pica, and A. Rago, Conformal vs confining scenario in SU(2) with adjoint fermions, Phys. Rev. D80 (2009) 074507, [arXiv: 0907.3896 ].

[23] LatKMI Collaboration, Y. Aoki, T. Aoyama, M. Kurachi, T. Maskawa, K.-i. Nagai, H. Ohki, E. Rinaldi, A. Shibata, K. Yamawaki, and T. Yamazaki, Light composite scalar in twelve-flavor QCD on the lattice, Phys.Rev.Lett. 111 (2013) 162001, [arXiv:1305.6006].

[24] LatKMI Collaboration, Y. Aoki, T. Aoyama, M. Kurachi, T. Maskawa, K.-i. Nagai, H. Ohki, E. Rinaldi, A. Shibata, K. Yamawaki, and T. Yamazaki, Light composite scalar in eight-flavor QCD on the lattice, Phys.Rev. D89 (2014) 111502, [arXiv: 1403.5000 ].

[25] LatKMI Collaboration, Y. Aoki, T. Aoyama, M. Kurachi, T. Maskawa, K. Miura, K.-i. Nagai, H. Ohki, E. Rinaldi, A. Shibata, K. Yamawaki, and T. Yamazaki, Lattice study of flavor-singlet scaler in large- $N_{f} Q C D, P o S$ KMI2013 (2015) 006.

[26] M. Luscher, Properties and uses of the Wilson flow in lattice QCD, JHEP 1008 (2010) 071, [arXiv:1006.4518]. 